\title{
Classification of Sensor Independent Point Cloud Data of Building Objects using Random Forests
}

\author{
Maarten Bassier ${ }^{1}$, Maarten Vergauwen ${ }^{1}$, \\ Bjorn Van Genechten ${ }^{2}$ \\ ${ }^{1}$ Dept. of Civil Engineering, TC Construction - Geomatics \\ KU Leuven \\ Belgium \\ ${ }^{2}$ Leica Geosystems, Hexagon, Brussels, Belgium \\ \{maarten.bassier, maarten.vergauwen\}@kuleuven.be \\ Bjorn.VanGenechten@leica-geosystems.com \\ http://iiw.kuleuven.be/onderzoek/geomatics \\ http://www.leica-geosystems.be/
}

\begin{abstract}
The Architectural, Engineering and Construction (AEC) industry is looking to integrate Building Information Modelling (BIM) for existing buildings. Currently these as-built models are created manually which is time-consuming. An important step in the automated Scan-to-BIM procedure is the interpretation and classification of point cloud data. This is computationally challenging due to the sheer size of point cloud data of an entire building. Additionally, the variety of objects makes classification problematic. Existing methods focus on specific sensors or environments to improve their results. The goal of this research is to provide a method that is sensor independent and labels entire buildings at once.

This paper presents a method to automatically identify structural elements for the purposes of Scan-to-BIM. More specifically, a Random Forests classifier is employed for the classification of the floors, ceilings, roofs, walls and beams. First, the point cloud is pre-segmented into planar primitives. This significantly reduces the data while maintaining accuracy. Both contextual and geometric features are used to describe the observed patches. By pre-
\end{abstract}


segmenting the data, more distinct features can be extracted from the input information. The algorithm is evaluated using realistic data of a wide variety of existing buildings including houses, school facilities, a factory, a castle and a church. The experiments prove that the proposed algorithm is capable of labelling structural elements with reported precisions of $85 \%$ and $87 \%$ recall in highly cluttered environments. In future work, the classified patches are processed by class-specific reconstruction algorithms to create BIM geometry. Keywords: Classification, Semantic Labelling, Scan-to-BIM, Pre-segmentation, Point Clouds, Building

\section{Introduction}

The implementation of Building Information Modelling (BIM) for existing buildings is gaining popularity. Experiencing the advantage of BIM for new constructions, the industry now looks to implement as-built BIM. The need for

5 resource efficiency, planning and communication have prompted stakeholders to adopt intelligent models that reflect the state of the asset as it was built to properly manage their data. These as-built models store an immense amount of information about a building at the varying stages of the construction's life cycle [1]. This metric and non-metric information allows the different parties 10 in the construction process to better operate, analyse and evaluate the asset [2]. As-built BIM is currently being employed for documentation, maintenance, quality control, etc. [3].

The production of BIM models with as-built conditions is labour intensive and error prone. The existing documentation of the asset is often sparse or non-existent. Moreover, it often does not match the as-design model of the building due to construction changes or renovations [4, 5]. Current procedures rely on dense spatial measurements for the modelling of the geometry. Typically, 3D point cloud data is acquired by 3D laser scanners or photogrammetry [6]. The automated reconstruction of BIM objects is still ongoing research [7]. 20 A key step in the workflow is the identification of observations of structural 
elements. Several machine learning algorithms have been proposed to classify building objects. However, these methods often rely on specific sensors or environments [8,9]. The goal of this research is to extend the existing approaches to be applicable to point cloud data of any source that includes both interior 25 and exterior environments of a wide variety of buildings.

This paper presents an automated solution for the classification of point cloud data for the purposes of as-built BIM. More specifically, structural elements such as floors, ceilings, roofs, walls and beams are automatically identified in existing structures. Typically, these structures have a wide variety of elements, are heavily cluttered and have problems with occlusion as depicted in Fig. 1. Machine learning techniques are proposed for the labelling of the elements. The scope of this research is focussed on the processing of metric information as it is the most consistent in point cloud data.

The remainder of this work is structured as follows. Section 2 presents a background in as-built modelling. In section 3 the related work is discussed. In Section 4 the methodology is presented. The test design and experimental results are proposed in Section 5. Finally, the conclusions are presented in Section 6

\section{Background}

The procedure of converting point cloud data to BIM geometry is referred to as Scan-to-BIM. Most automated workflows consists of three consecutive steps: Segmentation, classification and reconstruction. First, the point cloud is segmented into point clusters. It is considered as an instance of unsupervised pattern recognition and can be solved by several machine learning techniques [10]. Planar clusters are often proposed [11, 12] but other primitives are not excluded.

Second, each cluster is provided with a class label. This step, referred to as classification, is considered an instance of supervised learning that identi- 


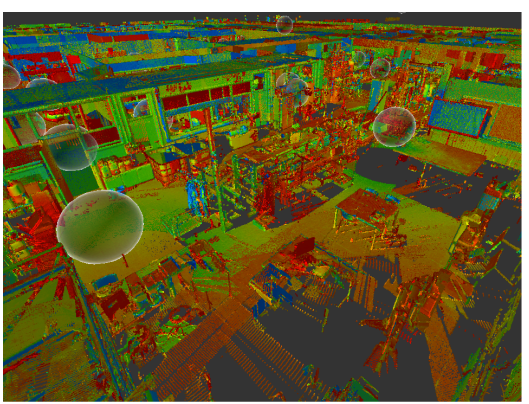

(a)

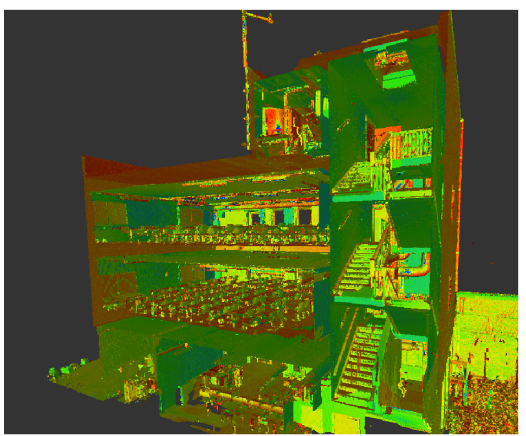

(c)

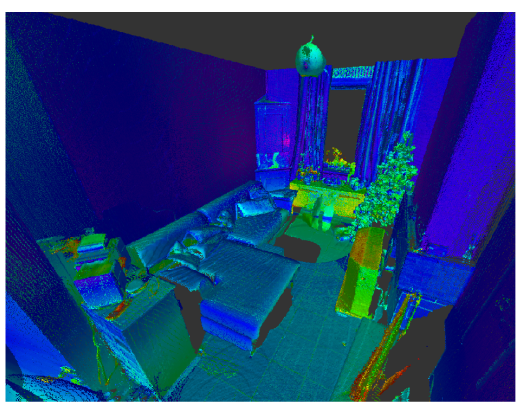

(b)

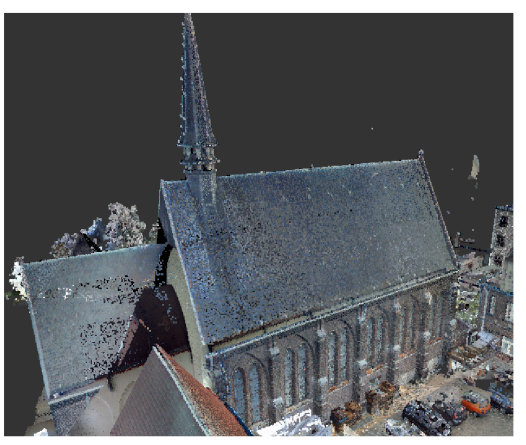

(d)

Figure 1: Example point clouds of structures used during testing: Chemical facility (a), house (b), multi-storey school building (c) and a church (d). 
fies the class of new observations given a set of explanatory variables known as features [10, 13]. Commonly in literature both geometric and contextual features of the observations are employed [14, 15]. Examples of local geometric features are the area, surface dimensions and orientation. Geometric contextual features may describe the similarity, proximity, coplanarity and orthogonality. The set of features, grouped in a feature vector, is processed by ${ }_{55}$ a pre-trained classification model to predict the labels. These functions are referred to as classifiers. Both heuristics and machine learning algorithms have been proposed for classification. Heuristic models are based on user defined rules in a certain structure. These rules require no training of the model parameters as they are intuitively set. While being very efficient, heuristics are typically case specific. Alternatively, machine learning algorithms are employed such as Discriminant Analysis (DA), Decision Trees, Support Vector Machines (SVM), Neural Networks (NN), Probabilistic Graphical Models (PGM), etc. [8, 12, 13, 16, 17, 18, 19]. The parameters of these models are learned from known observations. Machine learning methods often generalise better than heuristics but require extensive training data to work adequately. Currently, classification is used in a wide variety of applications such as navigation, object recognition and remote sensing.

In the third step, the labelled clusters are processed by class-specific reconstruction algorithms that create the BIM objects. Once the initial geometry has been constructed, the topology of the objects is adjusted to create a realistic BIM model. Afterwards, non-metric properties such as materials are added to the individual elements.

\section{Related Work}

Semantic labelling of building geometry has been a major research topic over the last decade. Both imagery and LIght Detection And Ranging (LIDAR) data are considered for the classification of structures. The terrestrial applications are split into the processing of indoor and outdoor scenery. Most indoor 
applications focus on the extraction of small scale objects for navigation and interaction purposes $[5,20,21,22,23,24]$. Outdoor applications often look to identify façades, windows, roads, etc. on a larger scale [9, 14, 25, 26, 27, 28, 29] Our approach is located in between as we look to label up to several hundreds of scans and generic point clouds of both indoor and outdoor scenery.

Closely aligned with our research is the work of Armeni et al. [30] and Xiong et al. [12, 31] who both proposed methods for the classification of structural elements of generic point cloud data for the purposes of $3 \mathrm{D}$ reconstruction. They label multiple scans of office and school facilities using machine learning techniques. We expand their approach by investigating whether pretrained models can be generalised for the classification of a wide variety of structures including castles, churches, factories, etc.

Related to the detection of structural elements is the classification of rooms in a building. Mura et al. [32], Ochmann et al. [32] and Ikehata et al. [33] consider the reconstruction to be a graph optimization problem. However, the assumption of closed rooms is only applicable for certain cases. In this research, the features do not specifically encode room information but describe certain object configurations such as the probability of a wall being located in between the floor and the ceiling.

Classification algorithms are often linked to a certain sensor set up or point cloud format [8, 15, 34, 35]. This allows the integration of additional information into the classification procedure. For instance, the sensors location provides cues for the positioning of certain objects. Valero et al. classifies individual scans captured by a Terrestrial Laser Scanner (TLS) for the segmentation of rooms [36]. However, some features are excluded as each scene is labelled individually. In our procedure, the input point cloud is considered as the sole entity and is labelled accordingly. This allows the use of multi-scene features such as the relation between walls in adjacent rooms.

Several researchers have presented results for the labelling of built envi- 
ronments using heuristics [37, 38, 39, 40, 41, 42]. However, to classify a wider variety of buildings, more complex models are considered. For instance, Support Vector Machines (SVM) have been a popular approach in the past few years [43, 44, 45]. SVM have been successfully implemented for the classification of both indoor and outdoor point cloud data. Non-linear models such as Decision Trees are also considered for the identification of building elements [12, 29, 46, 47]. These weak learners are often bootstrap aggregated or boosted to produce stronger classification algorithms [48]. In our research a similar Random Forests classifier is proposed for the semantic labelling. Additionally, Probabilistic Graphical Models (PGM) are considered $[8,15,28,49,50]$. By representing the data as nodes in a graph, probabilistic reasoning allows the likelihood maximisation of the labels of the nodes. Both implementations of Markov Random Fields (MRF) and Conditional Random Fields (CRF) have been presented for the classification of point cloud data [15, 34, 51, 52, 53, 54, 8]. Their ability to model pairwise and higher order edge potentials between the nodes increases the model performance and aids classification regularity. The pairwise terms smooth the labelling as most CRF implementations favour associative classification [28]. This is especially useful in point-wise classification methods that have weaker features [9]. Currently, Probabilistic Graphical Models are not implemented into our approach as we incorporate the contextual information such as the topology as unary terms.

Prior knowledge has also been proposed for semantic labelling of buildings. Existing plans or models significantly reduce the search area of object recognition algorithms and aid the scene understanding. Several methods have been proposed for model matching between the as-built and as-designed conditions [55, 56]. However, our approach does not rely on prior knowledge as it is typically scarce and error prone. 

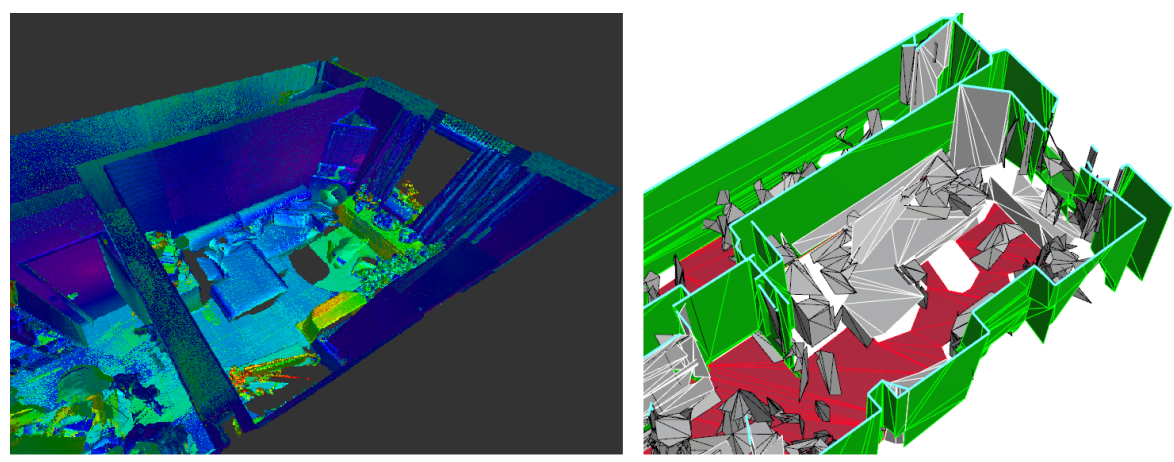

Figure 2: Pre-segmentation: Registered point cloud (Left) and the resulting planar patches (Right).

\subsection{Feature Extraction}

Distinct features are computed for each observed patch $s_{i}$ similar to Weinmann et al. [14]. Both geometric and contextual characteristics are exploited. The former encodes the object's individual geometric information such as shape, size and orientation. The latter encodes both associative and non-associative information in relation to a group of reference surfaces. In addition to the nearest neighbours, class-specific nearby patches are considered for the features. For instance, the repetitivity and frequent topological configurations of certain reference groups is captured. Table 1 summarizes the different types 
Table 1: List of all features calculated for each observed patch

\begin{tabular}{lc|lc}
\hline Local features & Count & Contextual features & Count \\
\hline Surface Area & 1 & Normal Similarity & 1 \\
Orientation & 2 & Coplanarity & 1 \\
Dimensions & 2 & Parallelity & 1 \\
& & Proximity & 4 \\
& & Topology & 5 \\
\hline
\end{tabular}

and the number of features used in the experiments. The context differs for the varying feature descriptors (Fig. 3). Both the immediate neighbourhood of the observed surface is exploited as well as larger reference groups. Additionally, several features only employ surfaces with specific characteristics such as orientation as context (Fig. 3 b,c). For instance, the vertical topology feature $D_{z}\left(u \in s_{i}, v \in s_{j}\right)$ evaluates the vertical distance between the observed surface $s_{i}$ and the large horizontal surfaces $s_{j}$ within its vicinity (Fig. $3 \mathrm{~b}$ ). The normal similarity feature computes $\vec{n}_{i} \cdot \vec{n}_{\text {large }}$ between the normal vector of the surface $\overrightarrow{n_{i}}$ and the normals of the nearby large surfaces $\vec{n}_{\text {large }}$. Coplanarity is defined as

$$
\operatorname{Coplanarity}\left(s_{i}, s_{j}\right)= \begin{cases}-1 & \overrightarrow{n_{i}} \cdot \overrightarrow{n_{j}}<\cos \theta_{t} \\ \left|\left(\overrightarrow{c_{i}}-\overrightarrow{c_{j}}\right) \cdot \overrightarrow{n_{i}}\right| & \text { else }\end{cases}
$$

where $\left|\left(\overrightarrow{c_{i}}-\overrightarrow{c_{j}}\right) \cdot \overrightarrow{n_{i}}\right|$ is the distance between centroids along the normal to the neighbouring parallel surfaces (Eq. 1). The parallellity is similar but with the reference surfaces conditioned to be directly in front of or behind of the observed surface. The proximity feature captures the repetitivity of certain object configurations. It is defined as the minimum distance $D_{\min }\left(u \in s_{i}, v \in s_{j}\right)$ between the boundary of the observed surface $s_{i}$ and a set of reference surfaces $s_{j}$. The following distances are evaluated: the vertical distance to the closest large horizontal surface above (1), the vertical distance to the closest large horizontal surface underneath (2), the distance to the closest large vertical surface 155 (3), and the number of connected surfaces (4). The topology features encode 


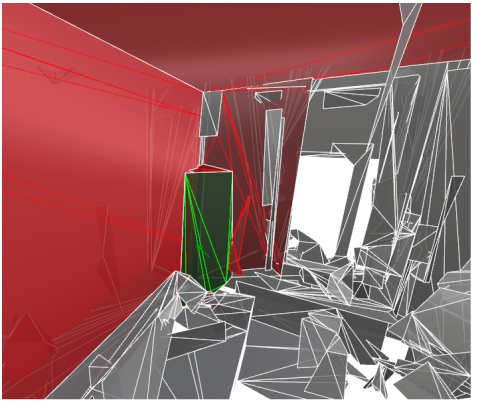

(a)

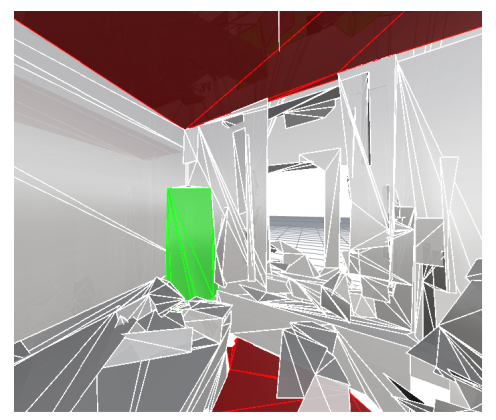

(c)

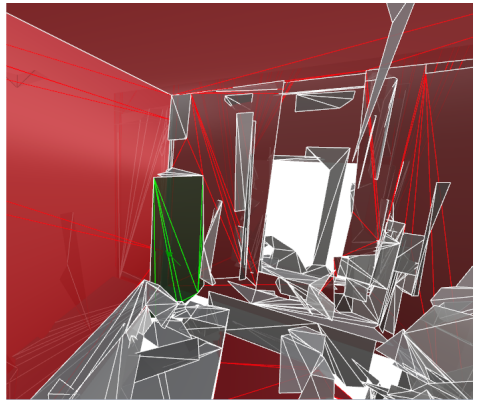

(b)

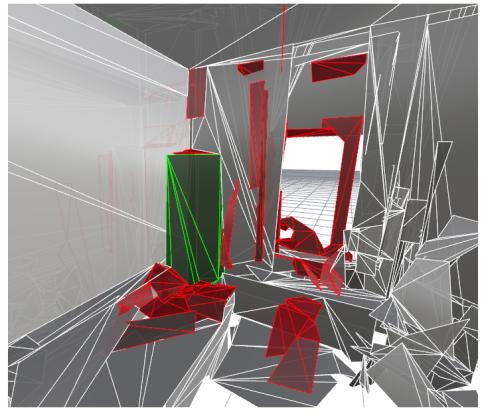

(d)

Figure 3: Visualisation of class specific neighbours: Conventional nearest neighbours (a), significantly large nearby surfaces (b), significantly large horizontal surfaces above/below (c) and nearby small surfaces $(\mathrm{d})$.

the presence of object configurations. These binary predictors serve as discriminative features between classes. For instance, a horizontal patch is more likely to be a ceiling when there are large horizontal patches directly above it. Five relations are observed describing the presence of horizontal and vertical surfaces: Small surfaces above (1) and below the patch (2) (Fig 4 a), the position of vertical patches (3) and the position of horizontal patches above (4) and below (5) (Fig 4 p). Finally, all features values are combined in feature vectors $\boldsymbol{x}=\left\{x_{1}, \ldots, x_{n}\right\}$ and are passed to the classifier for further processing.

\subsection{Model formulation}

Each observed surface is assigned a class label $y \in \boldsymbol{Y}=\left\{l 1, \ldots, l_{k}\right\}$ given the feature vectors $x$. The label prediction $p(y \mid x)$ is computed by a pre-trained Random Forests (RF) classifier similar to Munoz et al. [58], Vosselman et al. 

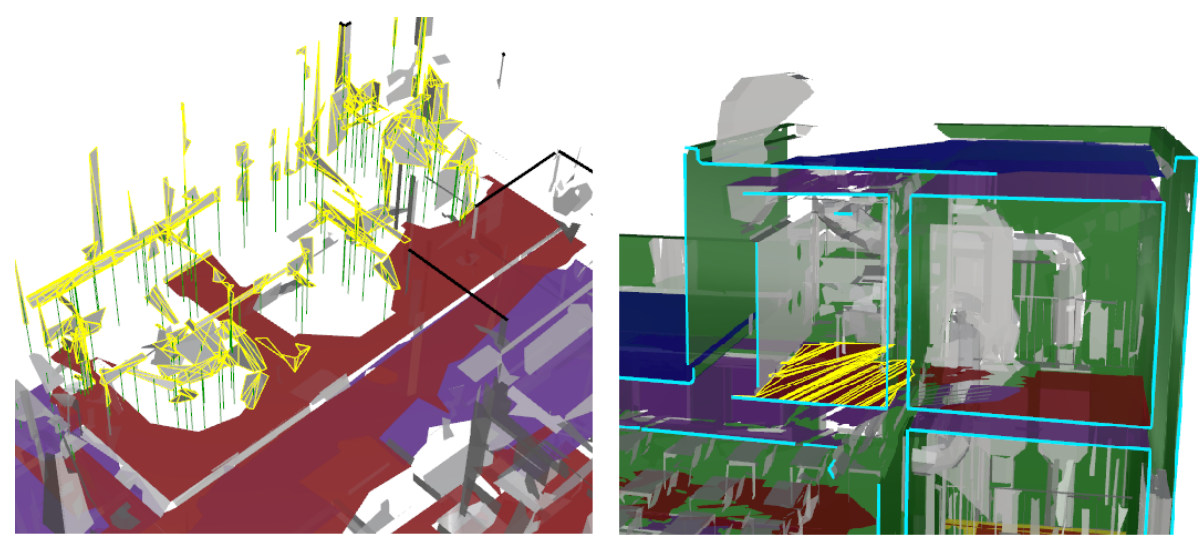

Figure 4: Representation topology predictors: Topology small surfaces (Left), evaluation topology nearby significantly large horizontal surfaces (Right).

[58], Niemeyer et al. [59], etc. [8, 54]. This classifier bootstrap-aggregates a number of weak learners [48]. The training data is copied into $M$ different bootstrapped data sets and a predictive discreet decision tree $y_{m}(\boldsymbol{x})$ is trained for each subset. Each tree consists of a series of binary splits that separate the input variables. The split is the result of the best fit partitioning of the feature vector space for the classes along one of the major axes. Typically, a single tree has the tendency to overfit. By bagging a large number of trees, the variance is significantly reduced which aids the generalisation of the model. Once the Random Forests classifier is trained, future observations are classified by a majority vote of the hypotheses of the decision trees.

The Random Forests model is trained using leave-p-out cross validation. Consecutively, p known observations are used for validation and the remaining observations are used for training. The final optimized model is achieved by averaging the model parameters. A regularization parameter $\lambda$ is introduced that penalises overfitting. The feature extraction and prediction algorithm are implemented in the Rhinoceros plug-in Grasshopper[60]. This intuitive procedural programming platform allows for flexible data processing and evaluation. The classified patches are exported to the Rhinoceros model space for validation and further processing. 


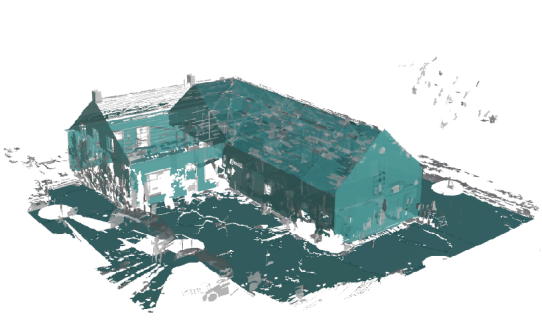

(a)

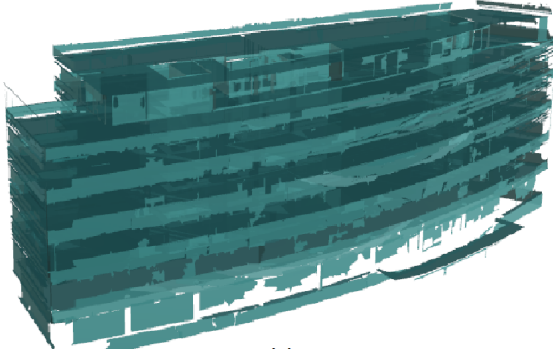

(c)

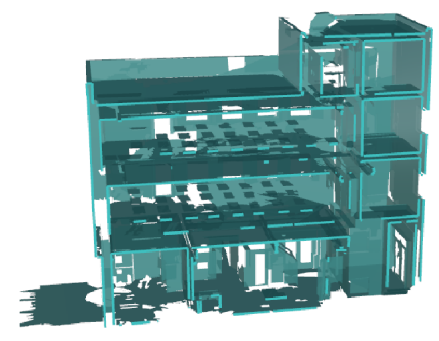

(e)

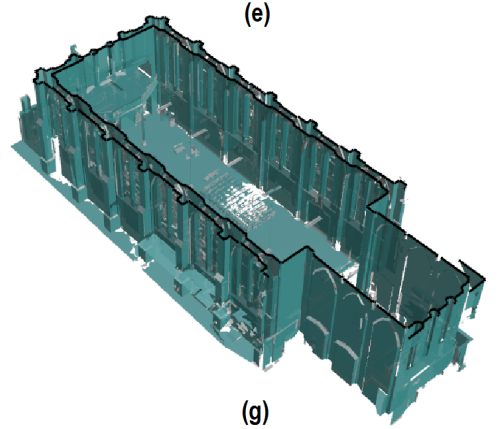

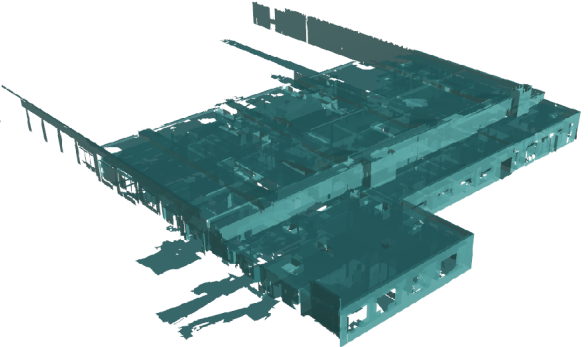

(b)

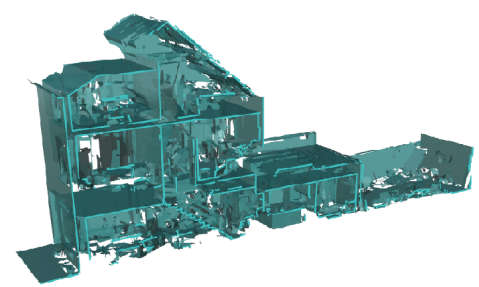

(d)

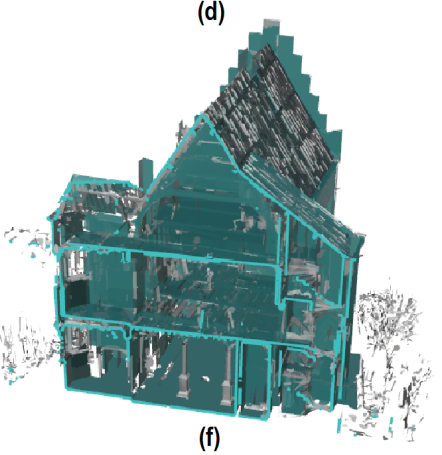

(f)

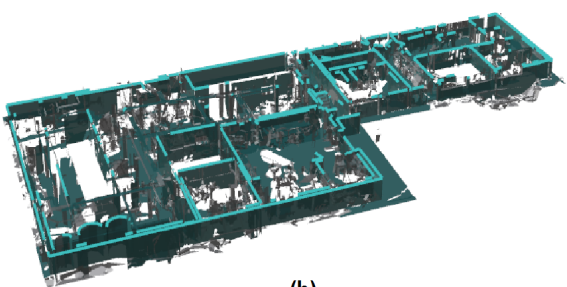

(h)

Figure 5: Example test cases with reconstructed surfaces: House 4000 surfaces (a), Campus 30000 surfaces (b), Office 26000 surfaces (c), Row house 1800 surfaces (d), Multi-storey office 3000 surfaces (e), heritage site 10000 (f), church 6500 surfaces (g) and chemical plant 15000 surfaces(h). 


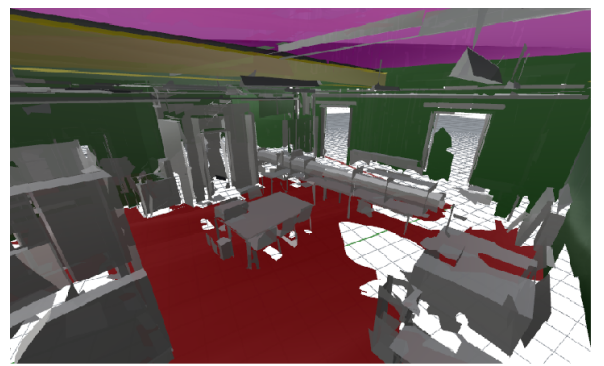

(a)

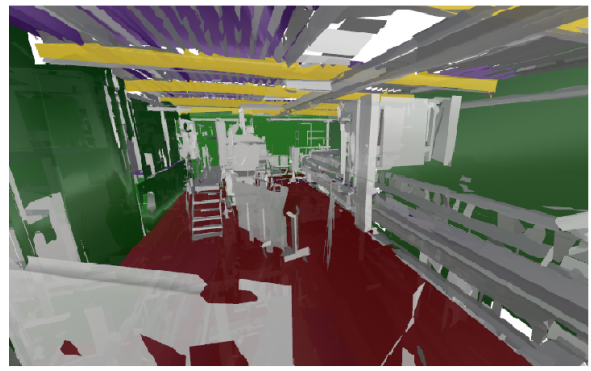

(c)

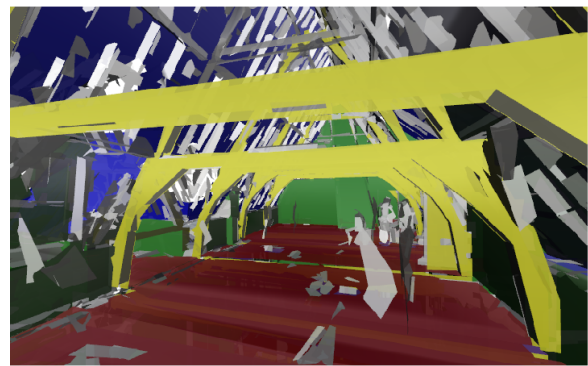

(e)

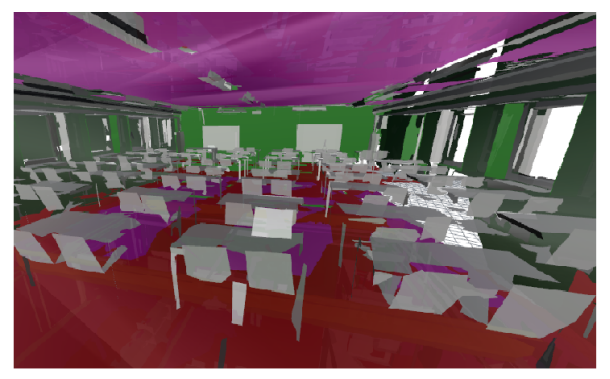

(b)

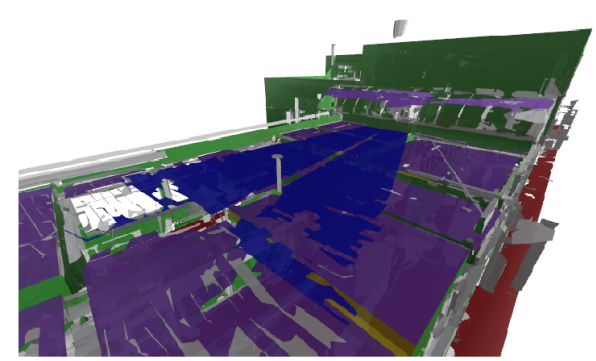

(d)

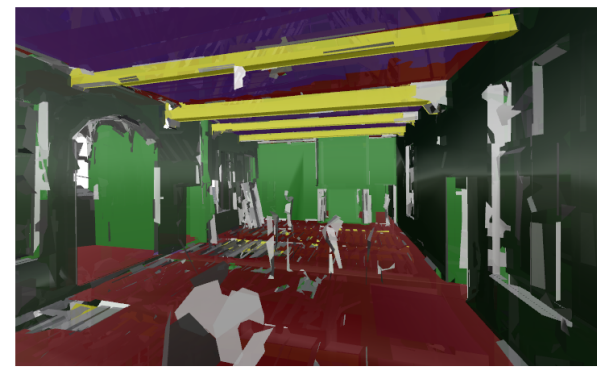

(f)

Figure 6: Example test data with ground truth: Laboratory (a), classroom (b), chemical plant (c), flat roof structure (d), heritage roof beam structure (e) and heritage room (f). Red=floors, purple $=$ ceilings, blue $=$ roofs, green $=$ walls, yellow $=$ beams and grey $=$ clutter. 


\section{Experiments}

The algorithm was tested on a wide variety of buildings. 10 structures including houses, offices, industrial buildings and churches were used for training and testing (Fig 5). The size of the projects varies between 300 million and 1,4 billion points and were acquired with different sensors such as Terrestrial Laser Scanners. The test sites were acquired under realistic conditions including clutter, occlusions, traffic, etc. The pre-segmentation was performed with the Aritmica's Pointfuse software [57]. The point clouds were subsampled with a $1 \mathrm{~cm}$ spacing and processed on an Intel ${ }^{\circledR}$ Core i7-4900MQ CPU @ 2.80Ghz with 4 cores and 4 hyperthreads and 32GB RAM. Over 90,000 surfaces were computed for the projects. On average, the patch creation had a standard deviation of $1 \mathrm{~mm}$ compared to the initial point clouds. A subset of the data was used for the experiments. Only the surfaces larger than $0.4 \mathrm{~m}^{2}$ were considered since the smaller surfaces mainly consisted of clutter and furniture pieces. The remaining 7000 surfaces were labelled manually and served as ground truth for the classification (Fig. 6). The available classes included floors, ceilings, roofs, walls, beams and clutter. All 17 predictors from table 1 were considered for the classification of the observations. The Random Forests classifier was trained with a $p=5$ cross-validation. In order to obtain an unbiased classification model, the training data set was balanced by sampling the same number of observations for each class.

\subsection{Performance}

The classification results are depicted in the confusion matrices in Fig.7 The average recall and precision is $87 \%$ and $85 \%$ respectively, resulting in an overall average accuracy of $86 \%$. This is very accurate given the large variety of buildings and objects that were evaluated. Typical objects such as floors, ceilings and walls were extracted with over $88 \%$ accuracy. This proves that while buildings have many unique objects and are heavily cluttered, their structural elements are reliably detected. 

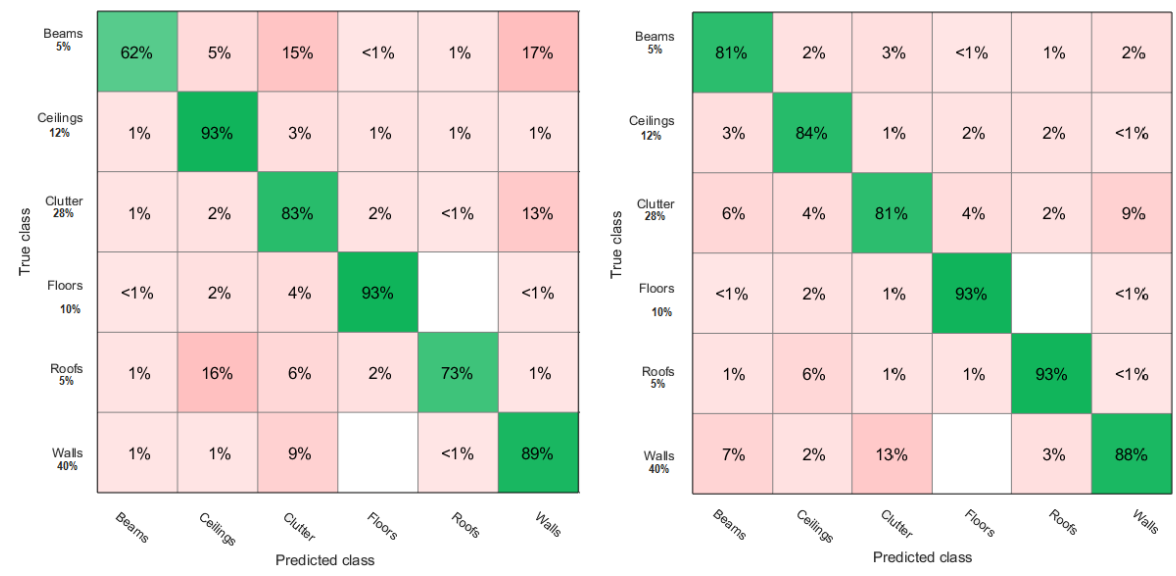

Figure 7: Classification results Random Forests classifier: Recall (Left), Precision (Right). The percentage defined under the 'True Class' is the amount of available test data.

Not-surprisingly, a large number of evaluated surfaces are objects for which the classes are not available (28\%). Combined with the small surfaces, over $90 \%$ of the pre-segmented patches are non-structural objects. However, the feature predictors are sufficiently distinct for the Random Forests classifier to properly differentiate the structural from the non-structural patches.

The confusion matrices show that the beams class underperforms (Fig. 7). This is expected due to the increased feature variance of this class. Beams have no obvious direction and are prone to occlusions. Increased confusion rates are observed between the walls and clutter classes as well as the ceiling and roof classes. The confusion rate of walls and clutter is approximately the same for both classes (avg. 10\%). This is mainly due to the misclassification of wall-like objects such as built-in closets and blackboards and actual wall objects such as niches and wall details (Fig 8 a,c,d \& e). In contrast, the confusion between the ceilings and roofs is only observed for the roofs (16\%). This is due to the fact that several data sets do not have roofs making the top ceilings harder to interpret (Fig 8b). Several misclassifications are due to our sensor independent approach. For instance, the ceiling in Fig $8 \mathrm{f}$ was misclassified as a floor as it was the most likely state given the context. Information regarding the position 


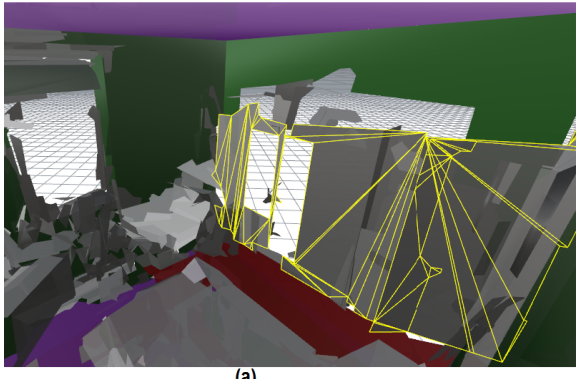

(a)

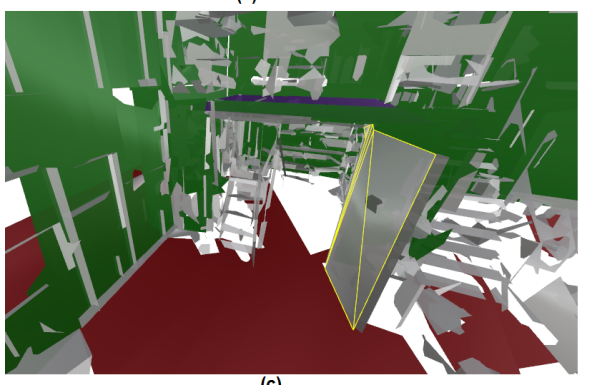

(c)

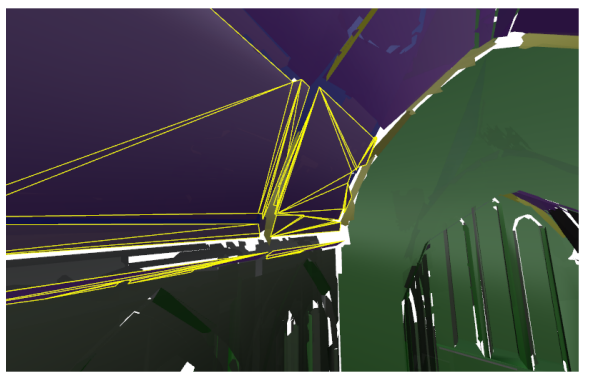

(e)
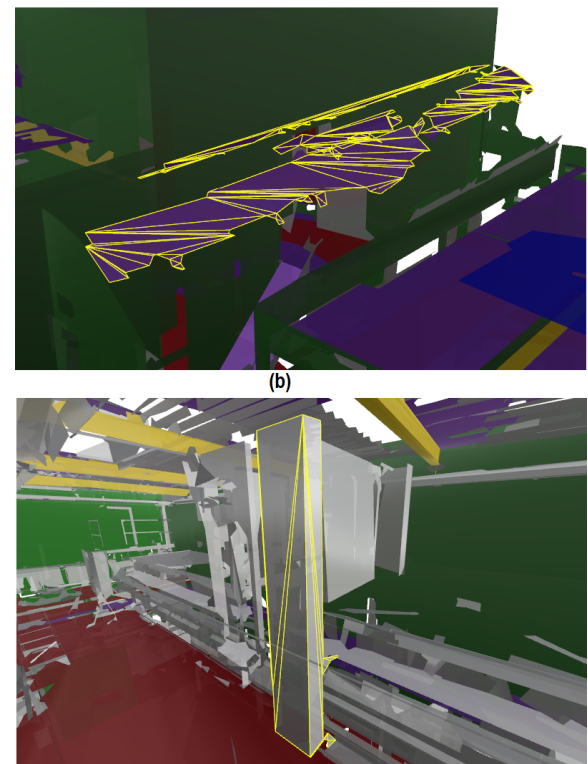

(d)

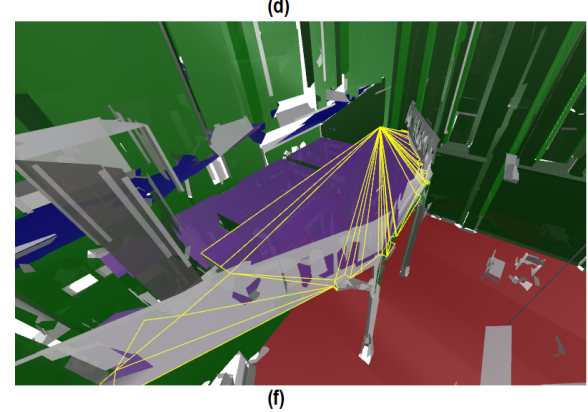

Figure 8: Several misclassification examples. Large closets, doors and machinery labelled as walls (a,c and d), unobstructed ceilings labelled as roofs (b), near vertical ceilings labelled as walls (e) and unobstructed ceilings labelled as floors (f). 
Table 2: Averaged classification results of Random Forests compared to K-Nearest Neighbours, multiceptron Neural Network, Linear Support Vectors Machines and Boosted Decision Trees.

\begin{tabular}{l|c|c|cccccc}
\multirow{2}{*}{ Classifier } & Training $[s]$ & AUC [\%] & \multicolumn{7}{|c}{ Recall/Precision [\%] } \\
& & & Beams & Ceilings & Clutter & Floors & Roofs & Walls \\
\hline Random Forests & 13.7 & $\mathbf{8 6}$ & $\mathbf{6 2 / 8 1}$ & $\mathbf{9 3 / 8 4}$ & $\mathbf{8 3 / 8 1}$ & $\mathbf{9 3 / 9 3}$ & $\mathbf{7 3 / 9 3}$ & $\mathbf{8 9 / 8 8}$ \\
KNN & 2.1 & 78.7 & $53 / 70$ & $74 / 70$ & $78 / 76$ & $82 / 72$ & $61 / 78$ & $\mathbf{8 5 / 8 7}$ \\
Neural Network & $\mathbf{1 . 2}$ & 69.6 & $61 / 29$ & $\mathbf{6 5 / 8 4}$ & $73 / 74$ & $87 / 78$ & $\mathbf{7 4 / 5 9}$ & $83 / 85$ \\
SVM & 15.8 & 74 & $13 / 51$ & $78 / 59$ & $71 / 71$ & $76 / 86$ & $52 / 69$ & $85 / 80$ \\
Boosted Trees & 14.4 & 77.8 & $27 / 70$ & $80 / 72$ & $76 / 69$ & $\mathbf{9 1 / 8 3}$ & $46 / 88$ & $85 / 84$
\end{tabular}

of the sensor would have avoided this misclassification but would make the approach dependent on a specific sensor set up.

\subsection{Comparison}

We compared the results of the Random Forests classifier with other common machine learning methods. Table 2 depicts the results of the model performance for K-Nearest Neighbours (KNN), a multiceptron Neural Network (NN), Support Vector Machines (SVM) and boosted decision trees. All models were tested with the same predictors and data as the proposed model. Both the recall and precision values were observed. From table 2 is derived that most classifiers had fast training speeds and were able to properly label the candidate data. This proves that the used predictors are both distinct and robust for the detection of structural elements in cluttered and noisy environments. When comparing Random Forests to the other classifiers, it is observed that the used model outperforms the other classifiers. When comparing against a Neural Network, a clear trade off is observed between speed and performance. However, since our approach focusses on post-processing applications, the training time is of lesser concern.

\section{Discussion and Conclusion}

In this paper, a method is presented for the automated classification of point cloud data of structural objects in buildings. More specifically, the data 
is pre-segmented and processed by machine learning algorithms to label the floors, ceilings, roofs, beams, walls and clutter in noisy and occluded environments. This step is crucial in the automated procedure of creating Building Information Modelling (BIM) objects from raw metric information.

By pre-segmenting the point cloud data, the amount of observations is drastically reduced while maintaining the metric accuracy. This allows for the processing of larger data sets and provides additional features. A Random Forests classifier is implemented that exploits both geometric and contextual features. A variable neighbourhood search is presented to encode various contextual relations. The results show that the proposed classifier is able to reliably label the pre-segmented patches given the feature observations. However, some classes underperform due to the large variance in feature values within the class. Classes such as beams consist of a wide variety of possible objects and suffer from occlusions. The results can be improved by narrowing down the possible objects of a single class by splitting the class into various subclasses such as pillar, beam, pipe, etc. Additionally, while the sensor independent approach generalises well, the lack of additional information such as the sensors position affects the model performance.

From the comparison to other classifiers is derived that most classification methods are capable of identifying the correct class of new observations given distinct and robust descriptors and a large number of known observations. The experiments prove that the feature values of observations in different buildings and configurations are similar and discriminative.

In future work, the method will be investigated further to improve the labelling performance. Additionally, More types of primitives will be considered along with appropriate features. This will enhance the current classification and allows for the processing of non-planar classes such as cylindrical beams and pipes as well as furniture. Also, research will be performed towards the integration of probabilistic graphical models to increase the methods perfor- 
mance.

\section{Acknowledgements}

Construction at the KU Leuven in Belgium is gratefully acknowledged. Additionally, the companies 3Dgeosolutions and Leica Geosystems are thanked for their data.

\section{References}

[1] BIMFORUM, Level of development specification, Tech. rep. (2016).

[2] M. D. Giudice, A. Osello, BIM for Cultural Heritage, International Archives of the Photogrammetry, Remote Sensing and Spatial Information Sciences XL (September) (2013) 225-229.

URL http: / /adsabs . harvard.edu/abs/2013ISPAr .XL5b .225D

295 [3] R. Volk, J. Stengel, F. Schultmann, Building Information Modeling (BIM) for existing buildings - Literature review and future needs, Automation in Construction 38 (2014) 109-127. doi : 10.1016/ j autcon.2013.10.023

URL http://dx.doi.org/10.1016/ j .autcon.2013.10.023

[4] I. Brilakis, M. Lourakis, R. Sacks, S. Savarese, S. Christodoulou, J. Teizer, A. Makhmalbaf, Toward automated generation of parametric BIMs based on hybrid video and laser scanning data, Advanced Engineering Informatics 24 (4) (2010) 456-465. doi:10.1016/ j . aei .2010.06.006

URL http://dx.doi.org/10.1016/j.aei.2010.06.006

[5] P. Tang, D. Huber, B. Akinci, R. Lipman, A. Lytle, Automatic reconstruc305 प

$$
\text { tion of as-built building information models from laser-scanned point }
$$
clouds: A review of related techniques, Automation in Construction 19 (7) (2010) 829-843. doi:10.1016/ j .autcon.2010.06.007.

a URL http://linkinghub.elsevier.com/retrieve/pii/ S0926580510000907 
[6] M. Bassier, M. Vergauwen, B. Van Genechten, Standalone Terres-

1 trial Laser Scanning for Efficiently Capturing Aec Buildings for As-Built Bim, ISPRS Annals of Photogrammetry, Remote Sensing and Spatial Information Sciences III-6 (July) (2016) 49-55. doi: 10.5194/isprsannals-III-6-49-2016 URL http://www.isprs-ann-photogramm-remote-sens-spatial-inf-sci . net/III-6/49/2016/isprs-annals-III-6-49-2016.pdf

[7] V. Patraucean, I. Armeni, M. Nahangi, J. Yeung, I. Brilakis, C. Haas, State of research in automatic as-built modelling, Advanced Engineering Informatics 29 (2015) 162-171. doi:10.1016/j .aei.2015.01.001.

[8] D. Wolf, J. Prankl, M. Vincze, Fast Semantic Segmentation of 3D Point Clouds using a Dense CRF with Learned Parameters, IEEE International Conference on Robotics and Automation (ICRA).

[9] T. Hackel, J. D. Wegner, K. Schindler, Joint Classification and Contour Extraction of Large 3D Point Clouds, ISPRS Journal of Photogrammetry and Remote Sensing (2017) 231-245.

[10] C. M. Bishop, Pattern Recognition and Machine Learning, Springer, Berlin, 2006.

[11] S. Barnea, S. Filin, Segmentation of terrestrial laser scanning data using geometry and image information, ISPRS Journal of Photogrammetry and Remote Sensing 76 (2013) 33-48. doi:10.1016/j.isprsjprs.2012.05.001.

a URL http://linkinghub.elsevier.com/retrieve/pii/ S0924271612000809

[12] X. Xiong, A. Adan, B. Akinci, D. Huber, Automatic creation of semantically rich 3D building models from laser scanner data, Automation in Construction 31 (2013) 325-337. do i : 10.1016/ j . autcon.2012.10.006.

a URL http://linkinghub.elsevier.com/retrieve/pii/ S0926580512001732 
[13] E. Alpaydin, Introduction to Machine Learning Second Edition, MIT Press, 2010.

[14] M. Weinmann, B. Jutzi, S. Hinz, C. Mallet, Semantic point cloud interpre-

1. tation based on optimal neighborhoods, relevant features and efficient classifiers, ISPRS Journal of Photogrammetry and Remote Sensing 105 (2015) 286-304. doi:10.1016/ j.isprsjprs.2015.01.016 URL http: / / dx.doi.org/10.1016/ j . isprs jprs.2015.01.016

[15] A. Anand, H. S. Koppula, T. Joachims, A. Saxena, Contextually guided semantic labeling and search for three-dimensional point clouds, The International Journal of Robotics Research 32 (1) (2012) 19-34. doi: 10.1177/0278364912461538. URL http: / / i jr.sagepub.com/cgi / doi / 10 . 1177/0278364912461538

[16] C. Sutton, A. Mccallum, An Introduction to Conditional Random Fields, (1) Foundations and Trends in Machine Learning 4 (4) (2011) 267-373. doi : $10.1561 / 2200000013$.

[17] J. Domke, Learning graphical model parameters with approximate marginal inference, IEEE Transactions on Pattern Analysis and Machine

a Intelligence 35 (10) (2013) 2454-2467. arXiv: 1301.3193, doi:10.1109/ TPAMI . 2013.31

[18] I. Armeni, O. Sener, A. R. Zamir, H. Jiang, I. Brilakis, M. Fischer, S. Savarese, 3D Semantic Parsing of Large-Scale Indoor Spaces, Proceedings of the IEEE International Conference on Computer Vision and Pattern Recognition 2016. doi:10.1109/CVPR.2016.170.

[19] M. Chizhova, D. Korovin, A. Gurianov, M. Brodovskii, A. Brunn, U. Stilla, घ T. Luhmann, Probabilistic Reconstruction of Orthodox Churches From Precision Point Clouds Using Bayesian Networks and Cellular Automata, ISPRS - International Archives of the Photogrammetry, Remote Sensing and Spatial Information Sciences XLII-2/W3 (February) (2017) 187-194. 
doi : 10.5194/isprs-archives-XLII-2-W3-187-2017.

URL http: / / www . int-arch-photogramm-remote-sens-spatial-inf-sci . net /XL I I - 2-W3/187/2017/

[20] E. Turner, A. Zakhor, Floor Plan Generation and Room Labeling of Indoor Environments from Laser Range Data, GRAPP, International Joint Conference on Computer Vision, Imaging and Computer Graphics Theory and Applications (2014) 1-12.

URL http://www.eecs.berkeley.edu/\{ \}elturner/papers/ 375 elturner\{_\}visigrapp\{_\}2014.pdf

[21] C. Cabo, C. Ordoñez, S. García-Cortés, J. Martínez, An algorithm for au-

1. tomatic detection of pole-like street furniture objects from Mobile Laser Scanner point clouds, ISPRS Journal of Photogrammetry and Remote Sensing 87 (2014) 47-56. doi:10.1016/ j. isprs jprs.2013.10.008

URL

http://linkinghub.elsevier.com/retrieve/pii/ S092427161300230X

[22] M. Previtali, M. Scaioni, L. Barazzetti, R. Brumana, A flexible method1. ology for outdoor/indoor building reconstruction from occluded point clouds, ISPRS Annals of Photogrammetry, Remote Sensing and Spatial Information Sciences II-3 (September) (2014) 119-126. doi : 10.5194/isprsannals-II-3-119-2014.

n URL http://www.isprs-ann-photogramm-remote-sens-spatial-inf-sci . net/II-3/119/2014/

[23] D. Maturana, S. Scherer, VoxNet: A 3D Convolutional Neural Network for Real-Time Object Recognition, IEEE/RSJ International Conference on Intelligent Robots and Systems (IROS) (2015) 922-928doi : 10.1109/ IR0S. 2015.7353481 .

[24] H. S. Koppula, A. Anand, T. Joachims, A. Saxena, Semantic Labeling of 3D Point Clouds for Indoor Scenes, in: Advances in neural information processing systems, 2011, pp. 1-9. 
[25] C. Dore, M. Murphy, Semi-Automated Generation of As-built BIM Facade Geometry from Laser and Image Data, Journal of Information Technology in Construction 19 (January) (2014) 20-46.

[26] B. Douillard, J. Underwood, N. Kuntz, V. Vlaskine, a. Quadros, P. Morton, a. Frenkel, On the segmentation of 3D LIDAR point clouds, 2011 IEEE International Conference on Robotics and Automation (2011) 27982805doi : 10.1109/ICRA.2011.5979818 URL http://ieeexplore.ieee.org/lpdocs/epic03/wrapper.htm? arnumber $=5979818$

[27] J. Huang, S. You, Point Cloud Labeling using 3D Convolutional Neural Network, International Conference on Pattern Recognition (September) (2016) 1-6. doi : 10.1109/ ICPR.2016.7900038

[28] A. Nguyen, B. Le, Contextual Labeling 3D Point Clouds with Conditional Random Fields Contextual Labeling 3D Point Clouds with, Asian Conference on Intelligent Information and Database Systems (October 2015). doi : $10.1007 / 978-3-319-05476-6$

[29] K. Liu, J. Boehm, Classification of Big Point Cloud Data Using Cloud Computing, ISPRS - International Archives of the Photogrammetry, Remote Sensing and Spatial Information Sciences XL-3/W3 (2015) 553-557. doi : 10.5194/isprsarchives-XL-3-W3-553-2015

I1 URL http://www. int-arch-photogramm-remote-sens-spatial-inf-sci .

net/XL-3-W3/553/2015/isprsarchives-XL-3-W3-553-2015.

a pdf $\{\%\} 5 C n h t t p: / / w w w . i n t-a r c h-p h o t o g r a m m-r e m o t e-s e n s-s p a t i a l-n f-s c i$. net/XL-3-W3/553/2015/

420 [30] I. Armeni, S. Sax, A. R. Zamir, S. Savarese, Joint 2D-3D-Semantic Data

a for Indoor Scene Understanding, arXiv:1702.01105 [cs.CV arXiv: 1702 . 01105

URL http://arxiv.org/abs/1702.01105 
[31] X. Xiong, D. Huber, Using Context to Create Semantic 3D Models of Indoor Environments, Procedings of the British Machine Vision Conference 2010 (2010) 45.1-45.11 doi : 10.5244/C.24.45. URL http://www.bmva.org/bmvc/2010/conference/paper45/index. html

[32] C. Mura, O. Mattausch, R. Pajarola, Piecewise-planar Reconstruction of Multi-room Interiors with Arbitrary Wall Arrangements, Computer Graphics Forum 35 (7) (2016) 179-188. doi : 10.1111/cgf. 13015.

[33] S. Ikehata, H. Yang, Y. Furukawa, Structured Indoor Modeling, Proceedings of the IEEE International Conference on Computer Vision (2015) 1540012doi : 10.1109/ICCV.2015.156.

[34] S. H. Khan, M. Bennamoun, F. Sohel, R. Togneri, I. Naseem, Integrating Geometrical Context for Semantic Labeling of Indoor Scenes using RGBD Images, International Journal of Computer Vision 117 (1) (2016) 1-20. doi: $10.1007 / \mathrm{s} 11263-015-0843-8$

[35] F. Husain, B. Dellen, C. Torras, Recognizing Point Clouds using Conditional Random Fields, 2014 22nd International Conference on Pattern Recognition (ICPR).

[36] E. Valero, A. Adán, C. Cerrada, Automatic method for building indoor boundary models from dense point clouds collected by laser scan-

口. ners, Sensors (Switzerland) 12 (12) (2012) 16099-16115. doi:10.3390/ $445 \quad$ S121216099

[37] M. Bassier, M. Vergauwen, B. Van Genechten, Automated Semantic Labelling of 3D Vector Models for Scan-to-BIM, 4th Annual International Conference on Architecture and Civil Engineering (ACE 2016) (2016) 93100 do i : $10.5176 / 2301-394 \mathrm{X}$

450 [38] V. Sanchez, A. Zakhor, Planar 3D modeling of building interiors from point cloud data, 2012 19th IEEE International Conference on Image 
Processing (2012) 1777-1780doi : 10.1109/ICIP.2012.6467225.

URL http://ieeexplore.ieee.org/lpdocs/epic03/wrapper.htm? arnumber $=6467225$

455 [39] H. Lin, J. Gao, Y. Zhou, G. Lu, M. Ye, C. Zhang, L. Liu, R. Yang, Semantic decomposition and reconstruction of residential scenes from LiDAR data, ACM Transactions on Graphics 32 (4) (2013) 1. doi: 10.1145/2461912. 2461969

URL http://dl.acm.org/citation.cfm?doid=2461912.2461969

[41] C. Wang, Y. K. Cho, C. Kim, Automatic BIM component exn traction from point clouds of existing buildings for sustainabil-

[42] H. Macher, T. Landes, P. Grussenmeyer, Point clouds segmentation as base

[44] B. Yang, Z. Dong, A shape-based segmentation method for mobile laser scanning point clouds, ISPRS Journal of Photogrammetry and Remote 
Sensing 81 (2013) 19-30. doi:10.1016/ j . isprsjprs.2013.04.002.

口

URL

http://linkinghub.elsevier.com/retrieve/pii/

S0924271613001020

[45] J. Yang, Z. K. Shi, Z. Y. Wu, Towards automatic generation of as-built BIM: 3D building facade modeling and material recognition from images, International Journal of Automation and Computing 13 (4) (2016) 338349. doi : 10.1007/s11633-016-0965-7.

[46] M. Gerke, J. Xiao, Fusion of airborne laserscanning point clouds and images for supervised and unsupervised scene classification, ISPRS Journal of Photogrammetry and Remote Sensing 87 (2014) 78-92. doi:10.1016/j.isprsjprs.2013.10.011.

(1) URL

http://linkinghub.elsevier.com/retrieve/pii/ S0924271613002335

[47] R. Guo, D. Hoiem, Labeling Complete Surfaces in Scene Understanding, International Journal of Computer Vision (October 2014) (2014) 172-187. doi : $10.1007 / \mathrm{s} 11263-014-0776-7$.

URL http:// link.springer.com/10.1007/s11263-014-0776-7

[48] L. Breiman, Random forests, Machine learning 45(1) (2001) 5-32. arXiv: arXiv: 1011.1669v3, doi:10.1017/CB09781107415324.004.

[49] R. B. Rusu, Z. C. Marton, N. Blodow, A. Holzbach, M. Beetz, Model-based and learned semantic object labeling in 3D point cloud maps of kitchen environments, IEEE/RSJ International Conference on Intelligent Robots and Systems, IROS (November) (2009) 3601-3608. doi:10.1109/IROS. 2009.5354759.

505 [50] D. Munoz, N. Vandapel, M. Hebert, Onboard Contextual Classification of 3-D Point Clouds with Learned High-order Markov Random, Robotics and Automation, 2009. ICRA'09. IEEE International Conference on. IEEE. 
[51] J. Niemeyer, F. Rottensteiner, U. Soergel, Contextual classification of lidar data and building object detection in urban areas, ISPRS

[52] Y. Zhang, S. Song, P. Tan, J. Xiao, PanoContext: A whole-room 3D context Journal of Photogrammetry and Remote Sensing 87 (2014) 152-165. doi:10.1016/j.isprsjprs.2013.11.001 URL http://linkinghub.elsevier.com/retrieve/pii/ S0924271613002359 model for panoramic scene understanding, Lecture Notes in Computer Science (including subseries Lecture Notes in Artificial Intelligence and Lecture Notes in Bioinformatics) 8694 LNCS (PART 6) (2014) 668-686. doi : 10.1007/978-3-319-10599-4_43.

[53] L. Landrieu, C. Mallet, M. Weinmann, Comparison of belief propagation and graph-cut approaches for contextual classification of 3D lidar point cloud data, IGARSS'2017.

[54] S. Guinard, L. Landrieu, Weakly supervised segmentation-aided classification of urban scenes from 3D LiDAR point clouds St ephane Guinard , Loic Landrieu To cite this version :, ISPRS Journal of Photogrammetry and Remote Sensing.

[55] H. Son, C. Kim, A. M. Asce, Y. K. Cho, M. Asce, Automated Schedule Updates Using As-Built Data and a 4D Building Information Model, Journal of Management in Engineering 33 (4) (2017) 1-13. do i : 10.1061/(ASCE) ME. 1943-5479.0000528.

[56] F. Bosché, Y. Turkan, C. Haas, Tracking the Built Status of MEP Works : Assessing the Value of a Scan-vs . -BIM System, Journal of Computing in Civil Engineering (March 2015). doi : 10.1016/ j . autcon .2014.05.014. URL http://dx.doi.org/10.1016/j.autcon.2014.05.014

[57] L. Arithmetica, Pointfuse (2015). URL http://pointfuse.com/ 
[58] D. Munoz, J. A. Bagnell, M. Hebert, Stacked Hierarchical Labeling, European Conference on Computer Vision (2010).

[59] J. Niemeyer, F. Rottensteiner, U. Soergel, Classification of Urban LiDAR data using Conditional Random Field and Random Forests, Proceedings of the JURSE (1) (2013) 139-142.

[60] R. McNeel, Rhinoceros 5, Tech. rep. (2015).

URL http://www.rhino3d.com 